(C) 2008 The Japan Society of Applied Physics

\title{
Readout Signals Enhancements of Subwavelength Recording Marks via Random Nanostructures
}

\author{
Tai Chi Chu, Wei-Chih LiU ${ }^{1}$, Din Ping TsaI ${ }^{2}$, and Yoshimasa Kawata \\ Department of Mechanical Engineering, Shizuoka University, Hamamatsu 432-8561, Japan \\ ${ }^{1}$ Department of Physics, National Taiwan Normal University, Taipei 11676, Taiwan \\ ${ }^{2}$ Department of Physics, National Taiwan University, Taipei 10617, Taiwan
}

(Received November 30, 2007; accepted March 6, 2008; published online July 18, 2008)

Super-resolution properties of super-resolution near-field structures (super-RENS) are analyzed by a simplified Fourier optics approach. Statistical averages of near-field optical disks with random nanostructures are calculated to realize their general behaviors. Averaged readout waveforms of near-field optical disks with random apertures are calculated numerically. The resolution of a near-field optical disk is determined by the size and shape of the random nanostructure.

[DOI: $10.1143 / \mathrm{JJAP} .47 .5767]$

KEYWORDS: super-RENS, near-field optical disk, Fourier optics, random structures, apertures

In order to obtain a higher recording density, the idea of using a masking layer to couple out subwavelength signal in near fields was developed. ${ }^{1)}$ Subsequently, the super-RENS was proposed to overcome the diffraction limit through internal nanostructures of disk. ${ }^{2)}$ Previous studies showed that various types of material such as $\mathrm{Sb}^{2,3)} \mathrm{AgO}_{x}{ }^{4)} \mathrm{PtO}_{x},{ }^{5)}$ and $\mathrm{ZnO}^{6)}$ are applied to near-field optical disks, and their working mechanisms can be related to internal random nanostructures of disks..$^{3,4,7-9)}$ The optical effects of nearfield optical disks with random nanostructures have been analyzed by a Fourier optics approach in previous works. ${ }^{9)}$ Averaged readout contrasts are calculated analytically and numerically. Results showed that resolutions of near-field optical disks with random apertures are determined by the size of apertures. To simplify the calculation, a singlefrequency grating is used as a periodic recording mark chain. However, realistic recording marks are more complicated. In this report, we calculate the readout waveforms of near-field optical disks with multi-frequency recording mark chains by angular spectrum representations. ${ }^{10,11)}$

The angular spectrum representation is used to decompose a complicated field into simple plane waves with wave vectors $\boldsymbol{k}$. The far field of a complicated near field can be derived from sum over all propagating plane waves, and far-field intensity distribution is the square of spectral amplitude. Figure 1 shows a simplified model of near-field optical disks with random nanostructures. The beam width of incident Gaussian wave is $a$, and the far-field intensity of the near-field optical disk is

$$
\begin{aligned}
I\left(k_{x}\right)= & \frac{1}{4 a \pi^{7 / 2}}\left[\int_{-\infty}^{\infty} \exp \left(\frac{-x^{\prime 2}}{a^{2}}\right) G\left(x^{\prime}\right) F\left(x^{\prime}\right) \exp \left(-i k_{x} x^{\prime}\right) d x^{\prime}\right] \\
& \times\left[\int_{-\infty}^{\infty} \exp \left(\frac{-x^{2}}{a^{2}}\right) G^{*}(x) F^{*}(x) \exp \left(i k_{x} x\right) d x\right],
\end{aligned}
$$

where $G(x)$ is the optical function of grating and $F(x)$ is the optical function of random nanostructures in the near-field optical disk. In conventional optical disks, the reflected signal variation induced by a mark edge represents logical " 1 " and the unchanged signal represents logical " 0 ". The logical signals can be represented by the flat part (logical " 0 ") and extreme variations of square wave (local " 1 "). Therefore, a square grating (as shown in Fig. 2) is used as the periodic mark chain.

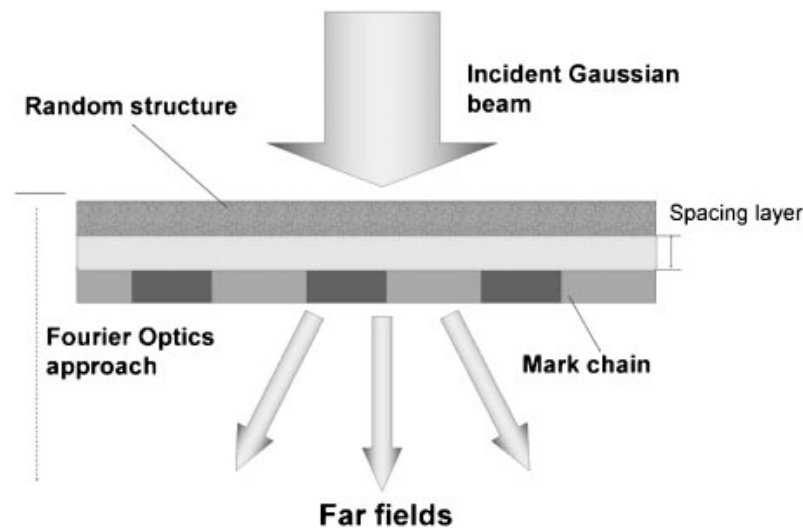

Fig. 1. Scheme of near-field optical disk with random nanostructures.

$$
\begin{aligned}
G(x)= & 1.2+0.6 \cos \left(\frac{\pi x}{L}\right)-0.2 \cos \left(\frac{3 \pi x}{L}\right) \\
& +0.1 \cos \left(\frac{5 \pi x}{L}\right)-0.07 \cos \left(\frac{7 \pi x}{L}\right),
\end{aligned}
$$

where $L$ indicates the mark length.

The averaged readout waveform of the grating with random nanostructures is derived numerically using eqs. (1) and (2). When the center of the laser beam shifts $s$ from the original position, the grating optical function shown in eq. (2) is changed to $G(x+s)$. It is noted that the result of a case with a particular random nanostructure is related to those specific random nanostructures. Thus, a statistical average is used to realize the general property of a near-field optical disk with random nanostructure. The optical function of random nanostructures is assumed to be the sum of Gaussian apertures at random positions

$$
F\left(x_{i}\right)=\sum_{i=1}^{N} \exp \left[-\left(x-x_{i}\right)^{2} / D^{2}\right],
$$

where $x_{i}$ is the uniformly distributed random position and $N$ is the amount of apertures. The far-field intensity variation $I\left(k_{x}, s\right)$ can be considered as signals of marks detected by apertures with random position $x_{i}$ and is a combination of cosine functions with various spatial frequencies $\left(g_{i}\right)$. Each component of a periodic signal [corresponding to cosine function in eq. (2)] contains a random phase that is related to the random variable $x_{i}$. In the averaging process, the periodic 


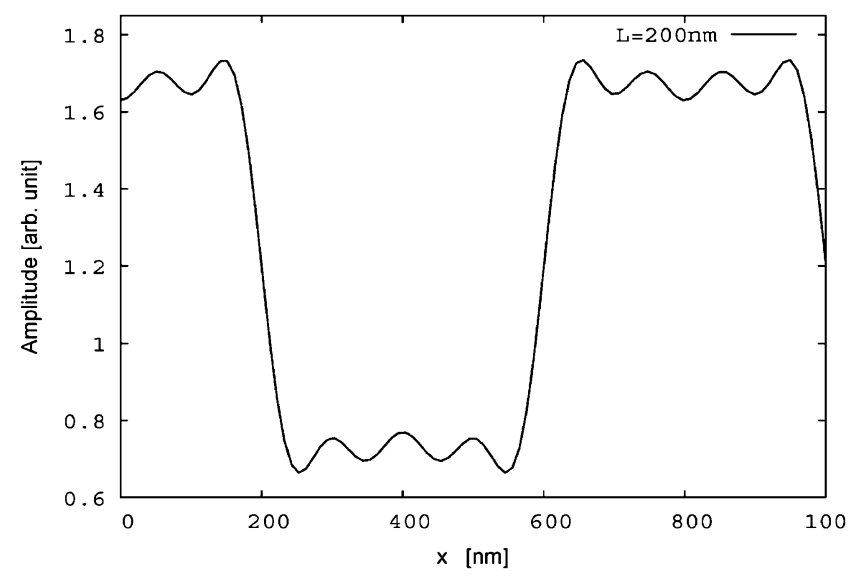

Fig. 2. Optical function of periodic recording marks for $L=400 \mathrm{~nm}$ case.

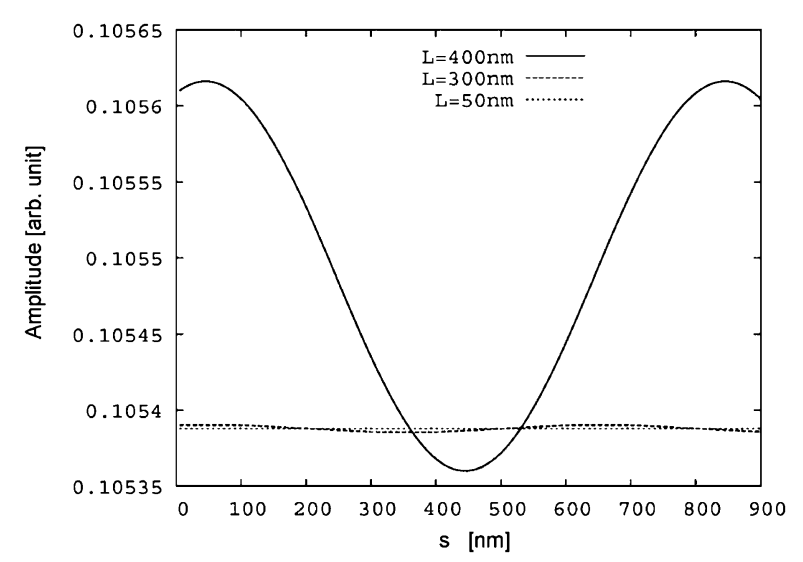

Fig. 3. Readout waveforms of cases without apertures (reference cases).

intensity (cosine function) is integrated over all random positions (random phases) and the averaged intensity is approaching a reference case (case with a uniform thin film). ${ }^{9)}$ In order to obtain realistic averaged signals, the phase shifts due to aperture positions should be eliminated. Thus, the calculated intensity variations (periodic signals) are shifted to correspond to the reference case (case without apertures), and 1000 realizations of random nanostructures with the same statistical nature are averaged to obtain the waveforms.

Using the conventional optical disk scheme, the wavelength $\lambda$ is $650 \mathrm{~nm}$ and numerical aperture (NA) is 0.6 . Figure 3 shows the waveforms of cases without apertures (reference case). Amplitudes of periodic signals decrease markedly when mark sizes are smaller than $300 \mathrm{~nm}$ because of diffraction limits. The averaged waveforms of cases with apertures are shown in Fig. 4. When aperture size $D$ is $7 \mathrm{~nm}$ [Fig. 4(a)], amplitudes of waveforms are almost constants for cases with 400, 300, and $50 \mathrm{~nm}$ mark sizes. Figure 4(b) shows results of $D=65 \mathrm{~nm}$ cases; amplitudes of waveforms are almost the same while mark sizes are larger than aperture sizes, and amplitudes decrease markedly for cases with $50 \mathrm{~nm}$ mark sizes.

The number of apertures $N$ in eq. (3) determines the transmittance of random nanostructure, and transmittances of cases shown in Fig. 4 are almost the same. The amounts of apertures for Fig. 4(a) $(D=7 \mathrm{~nm})$ and Fig. 4(b)

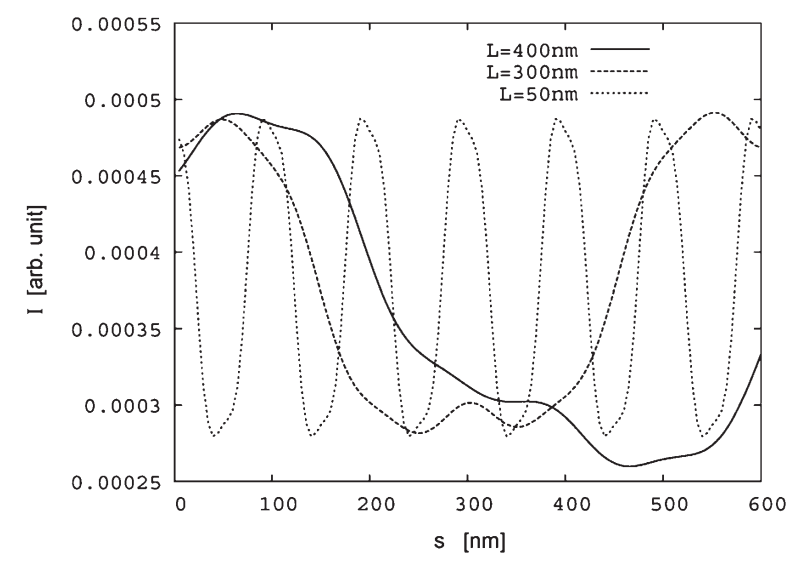

(a)

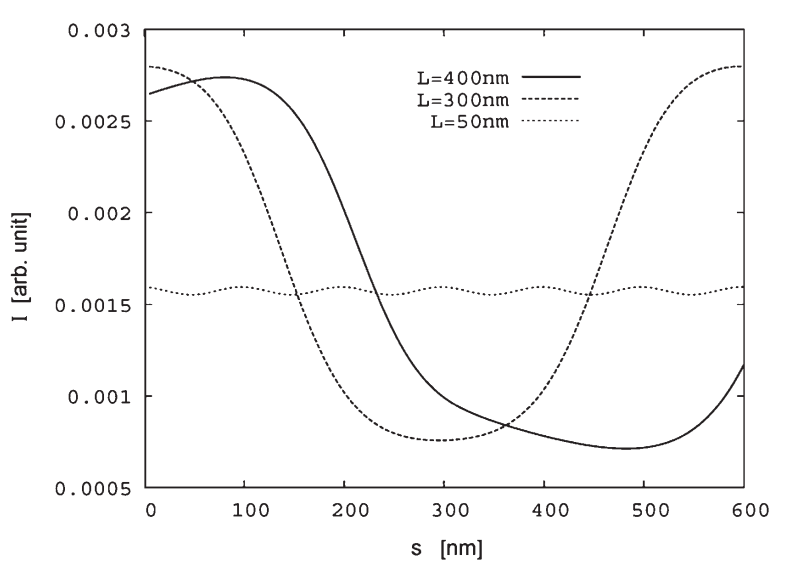

(b)

Fig. 4. Averaged readout waveforms of cases with random Gaussian apertures for (a) $D=7 \mathrm{~nm}$ and (b) $D=65 \mathrm{~nm}$.

$(D=65 \mathrm{~nm})$ are 16 and 2 , respectively. The total calculating regime is $4000 \mathrm{~nm}$, and the ratio of aperture coverage area to total calculation regime is about $5 \%$. When the ratio of aperture coverage area to total calculation regime reaches to $65 \%$, behaviors of waveforms with different mark sizes are similar to low ratio cases. Amplitudes of waveforms are obvious for cases with mark sizes larger than aperture sizes, and amplitudes diminish obviously for mark sizes smaller than aperture sizes.

In various types of super-RENS, many different random structures are found in the near-field active layer. For example, the random structures in $\mathrm{ZnO}_{x}$-type super-RENS ${ }^{12)}$ consisting of $\mathrm{ZnO}$ nanowires and appear similar to a combination of rectangular nanoapertures. Therefore, readout waveforms with random rectangular apertures are also calculated numerically and compared with cases with random Gaussian apertures. The optical function of the rectangular aperture is equal to 1 in the interval $\left[x_{i}-D, x_{i}+\right.$ $D]$ and 0 outside. The random number $x_{i}$ denotes the position of the aperture. Figure 5 shows the calculated waveforms of cases with rectangular apertures. Amounts of cases with 7 and $65 \mathrm{~nm}$ apertures sizes are 28 and 3, respectively. The ratio of aperture coverage area to total calculation regime is about $5 \%$. For $D=7 \mathrm{~nm}$ cases shown in Fig. 5(a), the amplitudes of waveforms are almost the same which correspond to the behaviors of Gaussian aperture cases [Fig. 4(a)]. Figure 5(b) shows the result of $D=65 \mathrm{~nm}$ case 


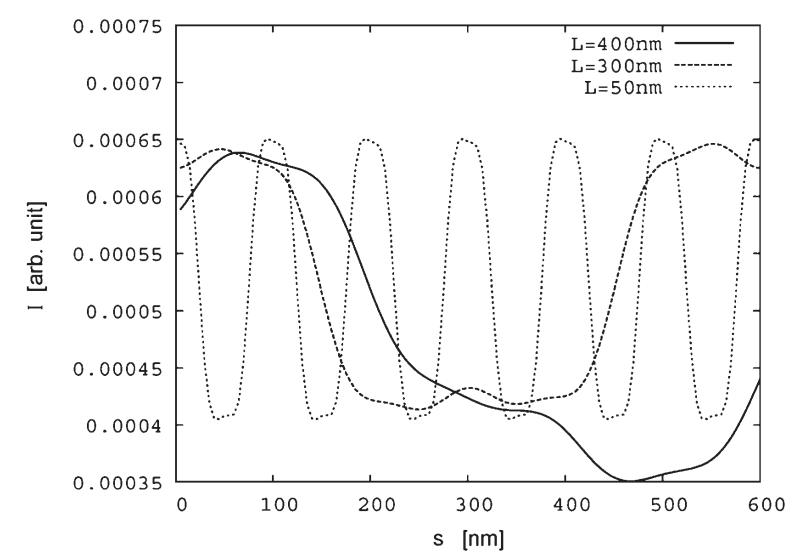

(a)

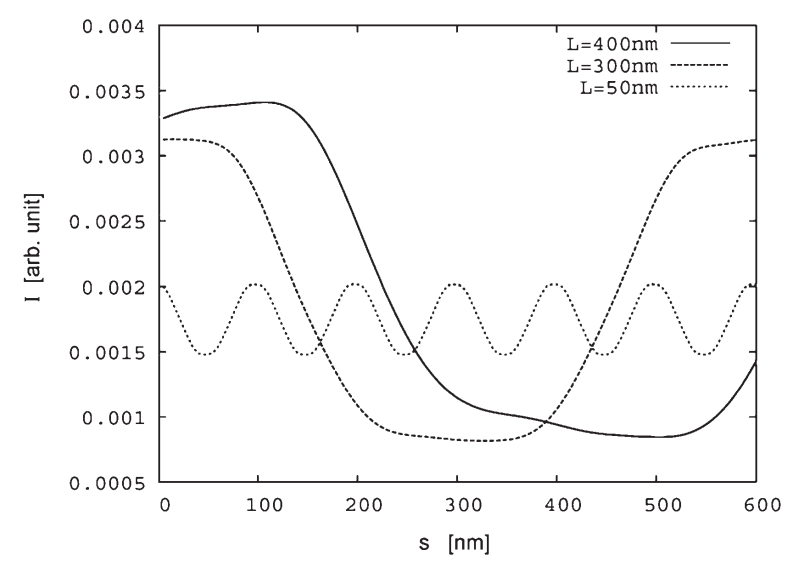

(b)

Fig. 5. Averaged readout waveforms of cases with rectangular Gaussian apertures for (a) $D=7 \mathrm{~nm}$ and (b) $D=65 \mathrm{~nm}$.

and the amplitude of the case with $50 \mathrm{~nm}$ marks is about $20 \%$ of that with $400 \mathrm{~nm}$ marks. Results show that the amplitudes of cases with rectangular apertures diminish more gradually than those of the cases with Gaussian apertures. This is because signals of recording marks are convoluted out by apertures, and apertures with broader Fourier spectra can couple out more evanescent signals. This idea is similar to a fiber probe in near-field optical technique. While the aperture on a probe apex is smaller (broader Fourier spectrum), the resolution of the probe is better. The Fourier spectrum of a rectangular aperture is $\operatorname{sinc}\left(k_{x} D\right)$ which is broader than that of a Gaussian aperture $\left[\exp \left(-k_{x}^{2} D^{2} / 4\right)\right]$ and has better resolution. When the ratio of aperture area to total calculation regime reaches to $65 \%$, waveforms with different mark sizes also show similar properties.
It is noticed that waveforms of larger mark size are more unsymmetrical than those of smaller mark size as shown in Figs. 4 and 5. This is because the periodic signal coupled out by each aperture has a phase difference related to its position, and the far-field signal is superposition of those periodic signals with different phases. When signals with random phases (due to aperture position) are summed over in average process, averaged waveforms diminish and approach to a reference case (symmetric). To get reasonable results, waveforms are shifted to match a reference case in our averaged process (as mentioned in the third paragraph). This shift of waveform means that random phases of signals are restricted in certain range. Interferences of high order components [in eq. (2)] with phases spreading in certain range induce unsymmetrical waveforms. For smaller recording marks (high spatial frequency), main contribution of far field is first order term $[\cos (\pi x / L)]$, and waveform is more symmetric due to fewer interference of high order component.

The general behaviors of simplified super-RENS are analyzed by a Fourier optics approach and using statistical averages. Random apertures in the near-field active layer work as apertures (or scatters) of fiber probes in near-field technologies, and sizes and shapes of apertures determine their resolutions. The Fourier spectrum of smaller aperture is broader which means more evanescent signals (high spatial frequency signal) can be convoluted out and the resolution is higher. Similarly, the Fourier spectrum of a rectangular aperture is broader than that of Gaussian apertures, and a rectangular aperture can couple out more evanescent signals. Our results reveal the super-resolution capabilities of optical disk with random nanostructures, and sizes and shapes of apertures determine their resolutions.

1) K. Yasuda, M. Ono, K. Aratani, A. Fukumoto, and M. Kaneko: Jpn. J. Appl. Phys. 32 (1993) 5210.

2) J. Tominaga, T. Nakano, and N. Atoda: Appl. Phys. Lett. 73 (1998) 2078.

3) D. P. Tsai and W. C. Lin: Appl. Phys. Lett. 77 (2000) 1413.

4) W.-C. Liu, C.-Y. Wen, K.-H. Chen, W. C. Lin, and D. P. Tsai: Appl. Phys. Lett. 78 (2001) 685.

5) T. Kikukawa, T. Nakano, T. Shima, and J. Tominaga: Appl. Phys. Lett. 81 (2002) 4697.

6) W. C. Lin, T. S. Kao, H. H. Chang, Y. H. Lin, Y. H. Fu, C. T. Wu, K. H. Chen, and D. P. Tsai: Jpn. J. Appl. Phys. 42 (2003) 1029.

7) T. C. Chu, W.-C. Liu, and D. P. Tsai: J. Scanning Microsc. 26 (2004) 102.

8) T. C. Chu, W.-C. Liu, and D. P. Tsai: Opt. Commun. 246 (2005) 561.

9) T. C. Chu, D. P. Tsai, and W.-C. Liu: Opt. Express 15 (2007) 12.

10) E. Wolf and M. Nieto-Vesperinas: J. Opt. Soc. Am. A 2 (1985) 886.

11) J. W. Goodman: Introduction to Fourier Optics (McGraw-Hill, New York, 1996) 2nd ed., p. 55.

12) T. Fukaya, D. Büchel, S. Shinbori, J. Tominaga, N. Atoda, D. P. Tsai, and W. C. Lin: J. Appl. Phys. 89 (2001) 6139. 